\title{
SEA ICE THICKNESS MEASUREMENT BY GROUND PENETRATING RADAR FOR GROUND TRUTH OF MICROWAVE REMOTE SENSING DATA
}

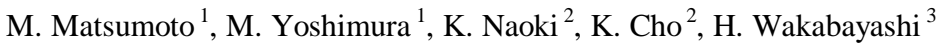 \\ ${ }^{1}$ PASCO CORPORATION, 2-8-10, Higashiyama, Meguro-ku, Tokyo, Japan - moatsy7605@pasco.co.jp \\ ${ }^{2}$ Tokai University, 2-28-4, Tomigaya, Shibuya-ku, Tokyo, Japan \\ ${ }^{3}$ Nihon University, 1 Nakagawara, Tokusada, Tamura, Koriyama, Japan
}

Commission III, WG III/2

KEY WORDS: Sea ice thickness measurement, Microwave remote sensing, Ground truth, Ground Penetrating Radar, Brackish lake, Finite-Difference Time-Domain method, Dielectric constant

\begin{abstract}
:
Observation of sea ice thickness is one of key issues to understand regional effect of global warming. One of approaches to monitor sea ice in large area is microwave remote sensing data analysis. However, ground truth must be necessary to discuss the effectivity of this kind of approach. The conventional method to acquire ground truth of ice thickness is drilling ice layer and directly measuring the thickness by a ruler. However, this method is destructive, time-consuming and limited spatial resolution. Although there are several methods to acquire ice thickness in non-destructive way, ground penetrating radar (GPR) can be effective solution because it can discriminate snow-ice and ice-sea water interface. In this paper, we carried out GPR measurement in Lake Saroma for relatively large area $(200 \mathrm{~m}$ by $300 \mathrm{~m}$, approximately) aiming to obtain grand truth for remote sensing data.. GPR survey was conducted at 5 locations in the area. The direct measurement was also conducted simultaneously in order to calibrate GPR data for thickness estimation and to validate the result. Although GPR Bscan image obtained from $600 \mathrm{MHz}$ contains the reflection which may come from a structure under snow, the origin of the reflection is not obvious. Therefore, further analysis and interpretation of the GPR image, such as numerical simulation, additional signal processing and use of $200 \mathrm{MHz}$ antenna, are required to move on thickness estimation.
\end{abstract}

\section{INTRODUCTION}

Observation of sea ice thickness is one of key issues to understand regional effect of global warming. One of approaches to monitor sea ice in large area is microwave remote sensing data analysis. For example, VV-to-HH backscattering ratio obtained by Synthetic Aperture Radar (SAR) system can be used to estimate ice thickness (Wakabayashi et al., 2004a). However, ground truth must be necessary to discuss the effectivity of this kind of approach.

The conventional method to acquire ground truth of ice thickness is drilling ice layer and directly measuring the thickness by a ruler. However, this method is destructive, timeconsuming and point information resulting in limited spatial resolution. Although there are several methods to acquire ice thickness in non-destructive way such as electromagnetic induction, sonar and so on, ground penetrating radar (GPR) can be effective solution because it can discriminate snow-ice and ice-sea water interface and comparative higher spatial resolution than the other methods (Liu et Al., 2014a). GPR which utilizes microwave is used to detect underground anomaly in subsurface (shallower than several meters) such as buried pipe, cavity, layer structures and even ice thickness (Tebaldini et al., 2016a). On Feb. 2017, we carried out GPR measurement in Lake Saroma, which is a brackish lake, in order to confirm utilization capability of GPR and estimated ice thickness (Matsumoto et al., 2017).

The goal of our study is to obtain ground truth for remote sensing data by using GPR. In this paper, we carried out GPR measurement in Lake Saroma for relatively large area $(200 \mathrm{~m}$ by $300 \mathrm{~m}$, approximately) aiming to obtain grand truth for remote sensing data. The methodology of thickness estimation and preliminary result will be presented.

\section{METHODOROGY}

\subsection{Ice thickness measurement by GPR}

Figure 1 shows skematic view of ice thickness measurement by GPR. GPR transmits microwave to underground and receives reflected wave caused by an interface of two different materials in terms of dielectric constant. From this figure, snow and ice thickness can be estimated by the time difference of arrival time of the reflections coming from snow-ice interface and icesea water interface. However, the propagation velocity, which is unknown and determined by the dielectric constant of material, is necessary in order to estimate these thicknesses. The propagation velocity $v$ is given by

$$
v=\frac{c}{\sqrt{\varepsilon_{r}}}
$$

where $\quad c=$ speed of electromagnetic wave in vacuum $\varepsilon_{\mathrm{r}}=$ dielectric constant of a medium

Our strategy to estimate snow and ice thickness is as following. Firstly we carry out GPR survey and acquire Bscan image which is a set of reflected signal along survey line. The direct measurement on the survey line is also conducted simultaneously. The precise position information of GPR antenna also should be acquired by RTK-GNSS in order to 
correlate Bscan image and result of the direct measurement. If the reflected signal from snow-ice interface and ice-sea water interface are observed, the velocity in the medium can be estimated by considering the time difference of arrival time of these reflections at the location where the direct measurement is conducted. Then, the snow and ice thickness can be estimated along the survey line by assuming the velocity in the medium is constant for all over the line.

\subsection{Numerical Simulation for Ice Thickness Measurement by FDTD method}

An electromagnetic field analysis was implemented in order to understand what kind of reflection we observe in ice thickness measurement and to confirm the strategy above. In this paper we used gprMax which is a free numerical GPR simulator based on FDTD (Finite-Difference Time-Domain) method (Warren et al., 2016a).

We assumed three layered model which consists of snow, ice and sea water, having layer thickness of $20 \mathrm{~cm}, 20 \mathrm{~cm}$ and $40 \mathrm{~cm}$, respectively. The dielectric constant of them is assumed as 6, 4 and 81 , and the conductivity is assumed $10^{-5} \mathrm{~S} / \mathrm{m}, 10^{-3} \mathrm{~S} / \mathrm{m}$, and $100 \mathrm{~S} / \mathrm{m}$, respectively by referring to a list of electric properties (Daniels, 2004). In this simulation, we assumed 600MHz GPR system by feeding first derivative of Gaussian pulse with central frequency of $600 \mathrm{MHz}$ as an electromagnetic source.

Figure 2 shows result of the simulation. Three main reflections can be seen, which correspond to 1) Air-snow interface and direct wave from transmitter, 2) Snow-ice interface, 3) Ice-sea water interface, respectively. Since the reflected signal has both of positive and negative peaks, an absolute value of analytic signal was calculated by using Hilbert transform in order to obtain a single peak. Then arrival time of the reflected signal was determined. From the time difference of these three reflections, which correspond to $t_{1}, t_{2}$, and $t_{3}$, the thickness of snow and ice were estimated as $19.41 \mathrm{~cm}$ and $20.03 \mathrm{~cm}$, respectively. The results are well agreed with actual thickness in the simulation model. Based on the simulation result, we summarised a flowchart to estimate ice and snow thickness as shown in Figure 3.

\section{FIELD EXPERIMENT}

\subsection{Test Field}

Field experiment was carried out in Lake Saroma, north east of Hokkaido, Japan, on $18^{\text {th }}$ to 22 of February 2018. Figure 4 shows sattlitte image of Lake Saroma taken by Sentinel-2 (Jan. $\left.18^{\text {th }}, 2018\right)$. Lake Saroma is facing to Sea of Okhotsk and connected by two inlets resulting in brackish lake. The lake is frozen over only winter season. Since the salinity of lake water is almost same as that of sea water, the ice of Lake Saroma can be considered as First Year ice of the sea. From Figure 4, the ice formed east and west side of the lake when the image was taken. The test field is located in the west side of the lake indicated by rectanguler.

Figure 5 (a) shows Satellite image around the test field. The area indicated by an arrow can be discriminated by the crack as shown in Figure 5 (b). Figure 5 (c) shows location of GPR survey lines in the right side of the crack area indicated by rectangular in Figure 5 (a). GPR survey was conducted at 5 locations in the area in order to cover relatively large area

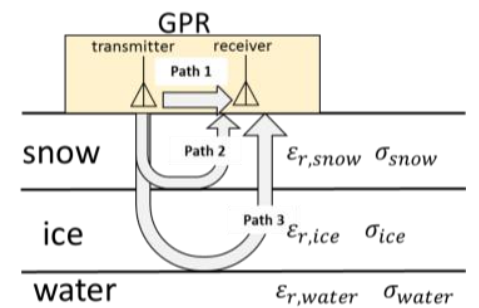

Figure 1. Skematic view of ice thickness measurement.

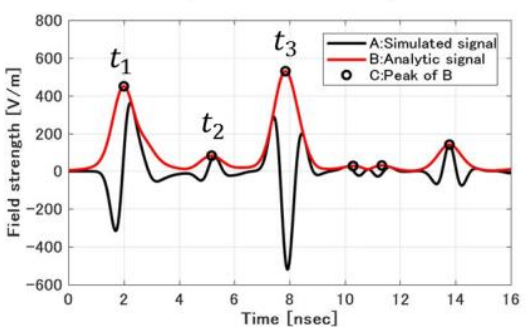

Figure 2. Reflected wave simulated by gprMax.

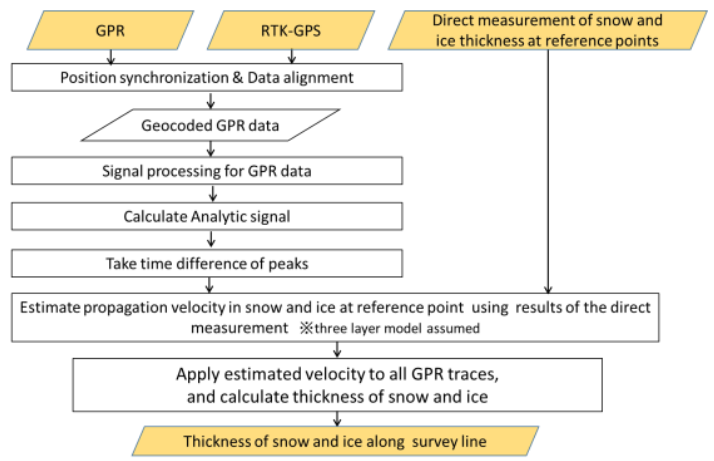

Figure 3. Flow chart of ice thickness measurement by GPR

(200m by $300 \mathrm{~m}$, approximately) aiming to obtain a grand truth for remote sensing data. The survey line on 2017 is also shown in the Figure.

\subsection{Measurement}

Figure 6 shows our GPR system used in the experiment. The radar unit is "RIS Hi-Mod", product of IDS GeoRadar s.r.l. HiMod can be operated in dual-frequency mode, $200 \mathrm{MHz}$ and $600 \mathrm{MHz}$. For the smooth movement of GPR, GPR is mounted on a thin plastic board to prevent the GPR from sinking in snow due to its weight. The hip chain encoder, which measures the length of a string pulled out, is equipped to GPR antenna, and then GPR can acquire the data at every $3 \mathrm{~cm}$ along the scan direction. Although the hip chain can acquire more correct positon data compared to a wheel type odometer in slip condition, positioning error can still arise because of the change of the tension of the string. Therefore we also equipped RTK-GNSS on the GPR antenna expecting to acquire correct position of GPR. The data collection of RTK-GNSS is every $0.1 \mathrm{sec}$.

After GPR survey, direct measurement along survey line was also conducted to calibrate snow and ice thickness acquired by GPR and to validate estimated results. As shown in Figure 5 (c), the direct measurement was carried out in No.1, No.2 and No.4 with interval of $3 \mathrm{~m}$. We drilled and measured thickness of snow and ice by ruler directly. 


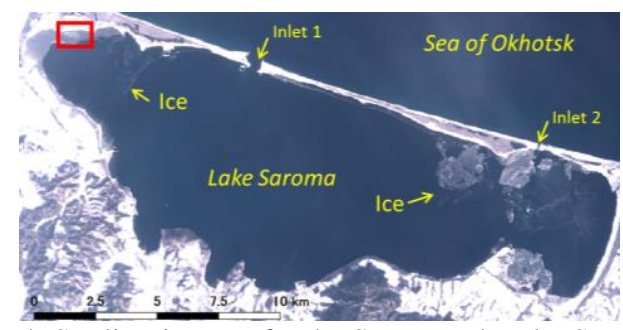

Figure 4. Sattlitte image of Lake Saroma taken by Sentinel-2, Jan. $18^{\text {th }}, 2018$. The test field is indicated by rectanguler.

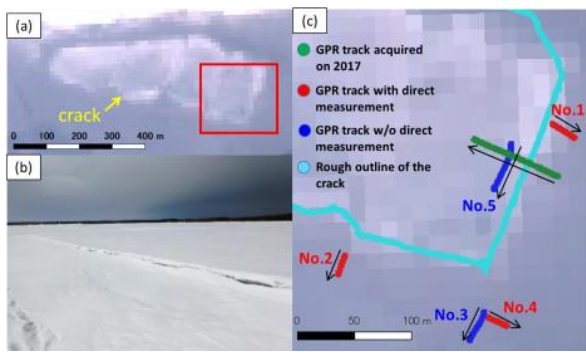

Figure 5. Test field located in the west side of the lake. (a) Sattellite image indicated by rectangular in Figure 4.

(b) Crack arround the area. (c) GPR survey line arround the rectanguler in (a). The arrows indicate scan direction.

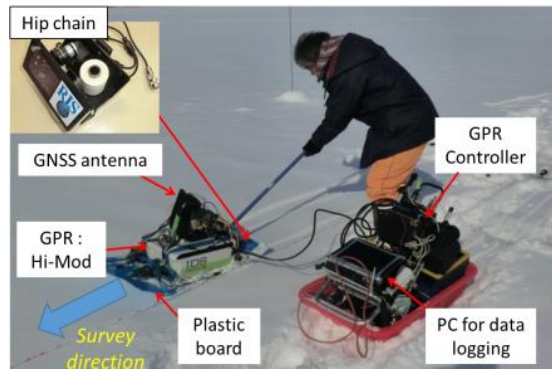

Figure 6 . The GPR system used in the experiment.

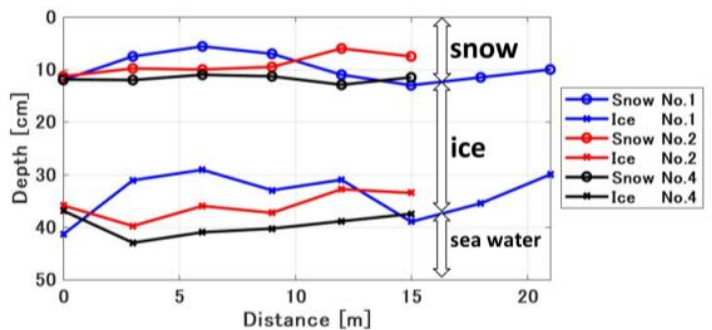

Figure 7. Snow and ice depth from snow surface obtained by the direct measurement along GPR survey line.

In this paper, we interpreted GPR Bscan image to prepare thickness estimation. The Bscan image was obtained by applying frequency band pass filter which suppresses DC bias and noise component, and gain control which enhances weak signal at deeper area.

\section{RESULTS}

\subsection{Snow and Ice Thickness along GPR Survey Line}

Figure 7 shows snow and ice depth from snow surface obtained by the direct measurement along GPR survey line No.1, No.2 and No.4. The average thickness of snow is $9.7 \mathrm{~cm}, 9.0 \mathrm{~cm}$ and $11.8 \mathrm{~cm}$ with standard deviation of $2.7 \mathrm{~cm}, 1.9 \mathrm{~cm}$ and $0.7 \mathrm{~cm}$ at No.1, No.2 and No.4, respectively. As for the ice thickness, the average of that is $24.1 \mathrm{~cm}, 26.9 \mathrm{~cm}$ and $27.8 \mathrm{~cm}$ with standard deviation of $3.2 \mathrm{~cm}, 1.9 \mathrm{~cm}$ and $2.5 \mathrm{~cm}$, respectively. The overall average and standard deviation of snow are $10.1 \mathrm{~cm}$ and $2.2 \mathrm{~cm}$, and those of ice are $26.0 \mathrm{~cm}$ and $3.0 \mathrm{~cm}$, respectively. When we carried out the experiment on 2017, the average thickness of snow and ice was $15.5 \mathrm{~cm}$ and $19.7 \mathrm{~cm}$, respectively. Therefore, the snow and ice was about $5 \mathrm{~cm}$ thinner and $6 \mathrm{~cm}$ thicker than 2017.

We also observed the condition between snow and ice layer. As for No.1, there was a mixture of snow and water above ice layer at distance of $9 \mathrm{~m}$ to $18 \mathrm{~m}$. As for No.2 and No.4, we found that snow just above ice layer is bit wet condition at all points.

\subsection{GPR Bscan Image}

Figure 8 shows GPR Bscan image obtained from $600 \mathrm{MHz}$ antenna for all survey line. Horizontal axis indicates survey distance which is directory obtained from hip chain encoder, and vertical axis indicates arrival time of the reflected signal. Note that aspect ratio of these figures are different depending on survey distance. As for distance axis, positioning error still exists caused by hip chain encoder. Roughly speaking, the error of distance of survey line is almost smaller than $-4.5 \%$ compared to GNSS results in this measurement. This means that if we carry out GPR survey with $20 \mathrm{~m}$ distance, the GPR records the data for only $19.1 \mathrm{~m}$. Therefore, position synchronization and GPR data alignment by GNSS is necessary to correlate GPR Bscan image and direct measurement. As for time axis, although GPR records the signal up to $80 \mathrm{nsec}$, the figures are shown up to $20 \mathrm{nsec}$. Since the reflection from ice sea water interface would arrive about 6nsec after the direct coupling arrives according to the simulation as shown in Figure $2,20 \mathrm{nsec}$ is enough long to see the result, because the direct coupling arrived about $5 \mathrm{nsec}$ in all Bscan images.

From the figures, the layer like structure can be observed in part of Figure 8 (a), (b), (c) and (e), and whole of (d) as indicated by arrow. These reflections are not completely horizontal but vertically changes. Although this implies that these signals can be caused by some structures under the snow, it is not obvious where the reflection comes from at this moment. Since the average snow and ice thickness was about $5 \mathrm{~cm}$ thinner and $6 \mathrm{~cm}$ thicker than 2017, we may need to adjust the numerical simulation model in order to figure out origin of these reflections.

Besides, horizontal reflection can be seen at $9 \mathrm{nsec}$ and $18 \mathrm{nsec}$ in Figure 8 (a), (b) and (c). These may not be a reflection from a layer structure but something caused by antenna coupling or reflection from snow surface, because these reflection is horizontally constant. These reflections should be eliminated because they may cover the weak signal arriving at same time. Average subtraction, which subtracts mean trace of Bscan image from each trace, may be effective for this purpose.

Figure 9 shows Bscan image obtained from $200 \mathrm{MHz}$ antenna at survey line of No.4. The layer like structure indicated by an arrow can be clearly seen in the image. Although lower frequency antenna cannot detect small changes due to low resolution image, it may be better to get perspective of the structure. Therefore the use of $200 \mathrm{MHz}$ data also should be considered. 


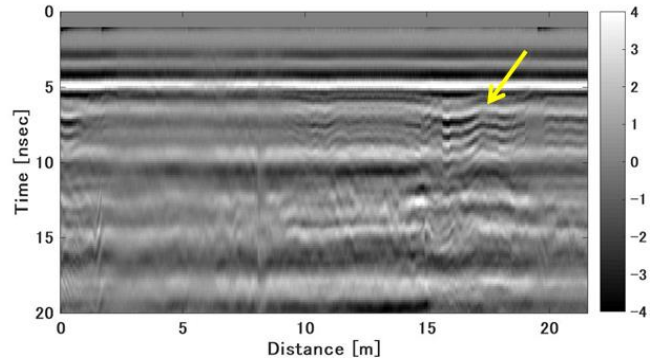

(a) No.1

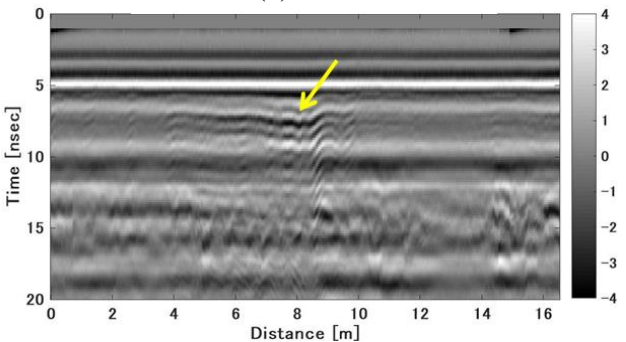

(b) No.2

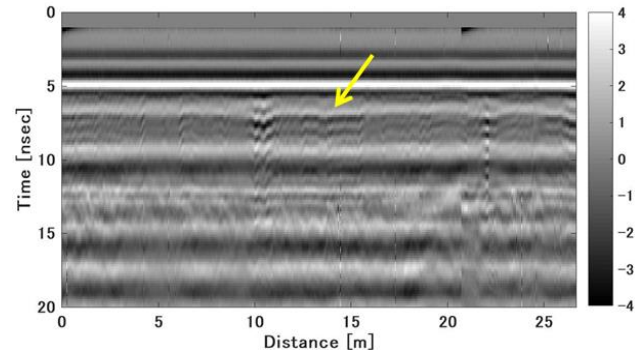

(c) No.3

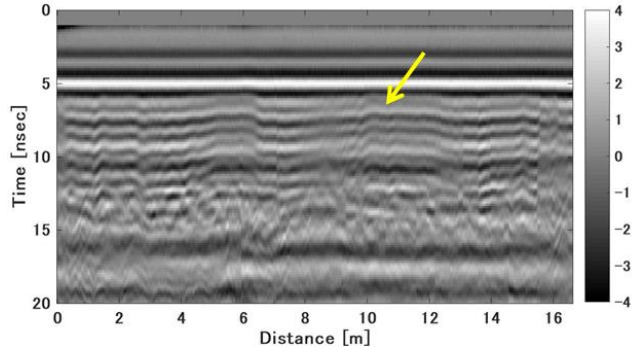

(d) No.4

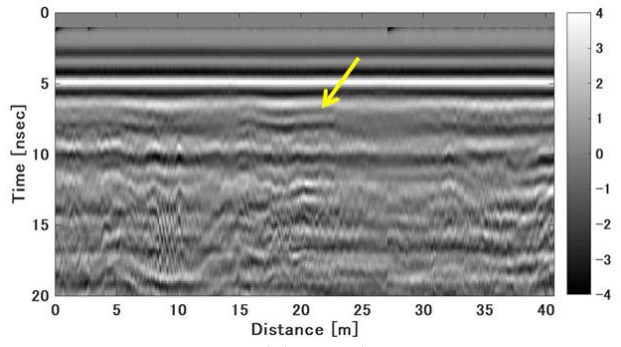

(e) No.5

Figure 8. Bscan image obtained from 600MHz antenna.

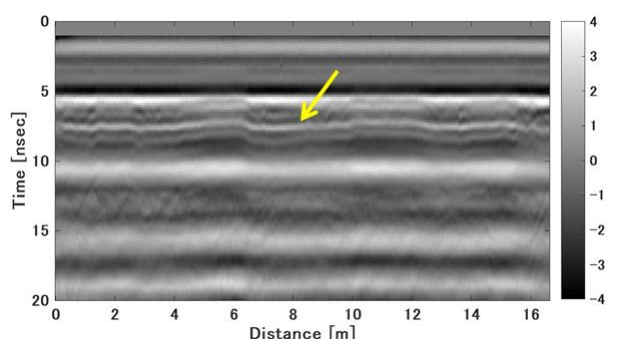

Figure 9. Bscan Image obtained from $200 \mathrm{MHz}$ antenna at survey line of No.4.

\section{CONCLUSION}

In this paper, we carried out GPR measurement in Lake Saroma for relatively large area $(200 \mathrm{~m}$ by $300 \mathrm{~m}$, approximately) aiming to obtain grand truth for remote sensing data. GPR survey was conducted at 5 locations in the area. The direct measurement was also conducted simultaneously in order to calibrate GPR data for thickness estimation and to validate the result. Although GPR Bscan image obtained from $600 \mathrm{MHz}$ contains the reflection which may come from a structure under snow, the origin of the reflection is not obvious. Therefore, further analysis and interpretation of the GPR image, such as numerical simulation, additional signal processing and use of $200 \mathrm{MHz}$ antenna, are required to move on thickness estimation. Looking at all Bscan images, different survey lines give different image features. This difference may reflect the local difference of snow and ice condition. Further consideration how associate the GPR data with remote sensing data in terms of spatial and time scale will be a future work.

\section{ACKNOWLEDGEMENTS}

The authors acknowledge Mr. T. Sato for his support in the field measurement.

\section{REFERENCES}

Daniels, D.J. (Ed.), 2004. Ground Penetrating Radar 2nd Edition. The Institution of Engineering and Technology, London.

Liu, H., Takahashi, K., Sato, M., 2014a. Measurement of Dielectric Permittivity and Thickness of Snow and Ice on a Brackish Lagoon Using GPR. IEEE Journal of Selected Topics in Applied Earth Observations and Remote sensing, Vol.7, No.3. pp. 820-827.

Matsumoto, M., Yoshimura, M., Naoki, K., Cho, K., 2017. Preliminary Experiment of Sea Ice Thickness Measurement by Ground Penetrating Radar. The 14th workshop on subsurface electromagnetic measurement, IEICE Technical Report, vol. 117 , no. 222, SANE2017-52, pp. 43-48.

Tebaldini, .S, Nagler, T., Rott, H., Heilig, A. 2016a. Imaging the Internal Structure of an Alpine Glacier via L-Band Airborne SAR Tomography. IEEE Transactions on Geoscience and Remote sensing, Vol.54, No.12. pp. 7197-7209

Wakabayashi, H., Matsuoka, T., Nakamura, K., Nishio, F., 2004a. Polarimetric Characteristics of Sea Ice in the Sea of Okhotsk Observed by Airborne L-Band SAR. IEEE Transaction on Geoscience and Remote Sensing, Vol.42, No.11. pp. 2412-2425.

Warren, C., Giannopoulos, A., Giannakis, I., 2016a. gprMax: open source software to simulate electromagnetic wave propagation for Ground Penetrating Radar, Computer Physics Communications, Vol.209, p.163-170. 